\title{
QUANTUM DISONTENTS, OR WHAT IS WRONG WITH OUR SCIENCE PRACTICE
}

\author{
Gennady Shkliarevsky \\ Bard College
}

\begin{abstract}
For more than eighty years the standard interpretation (SI) has dominated quantum physics. Perspectives that have tried to challenge this domination have been remarkably unsuccessful. As a result, quantum theory (QT) has remained remarkably stagnant. The article offers a critical examination of SI and provides an explanation for its continued domination. It also uses Bohmian mechanics-a theoretical perspective advanced by American physicist David Bohm-as a case study for why alternative interpretations have failed to displace SI. The article sees the main reason for the failure to achieve much progress beyond SI in the unresolved philosophical problem of subject-object relation that continues to plague our study of physics. The article sketches a path to a possible solution and outlines a new science practice that this solution will require.
\end{abstract}

Key words: standard interpretation, Bohmian mechanics, quantum uncertainty, determinism, subject-object relations, systems theory.

\section{Introduction}

For more than eighty years quantum theory (QT) has dominated the science of physics. It has been a rich source of ideas and perspectives that have shaped many spheres of our knowledge from physics to chemistry, computer science, information, chemistry, biology, psychology, philosophy, and even social sciences. One cannot think of our civilization without thinking about quantum theory.

In the course of its history QT has experienced many changes. Indeed, QT as we know it today is in many ways very different from, and much richer than its original formulation in the early $20^{\text {th }}$ century. Yet its fundamental features remain unchanged. Today the basic premises, definitive experiments, and main conclusions of QT are still the same as they were at the time of its inception; its controversies and paradoxes still provoke hot debates. The most important and controversial problems of QT are still awaiting their solutions.

For much of its history, the dominant trend in QT has been and still is its standard interpretation (SI). SI has demonstrated a remarkable and enviable longevity. It has successfully withstood the onslaught of time that has devastated and rendered obsolete many other theories. SI, by contrast, is as influential today as it was when it was first formulated, with few competitors to take its place. 
Many researchers feel that this remarkable staying power proves that SI is fundamental correct. There are, however, others-and their number is growingwho are very resentful of the fact that physics has not been able to step much beyond the horizon circumscribed by SI. They see its continued domination as the principal cause of what they see as stagnation in our physical science. In their view, SI has become an obstacle to the evolution of our knowledge about physical reality and a hindrance to its progress.

Although SI has no shortage of contenders, none of them has so far managed to displace its grand edifice. This fact in itself is an interesting problem that begs for an explanation. One cannot attribute the longevity of SI exclusively to its success. There have been many successful theories in the past that nevertheless have become obsolete and faded into obscurity. Why has SI so far escaped this fate?

\section{The Standard Interpretation and Its Critique}

The rise of SI has marked a significant progress in our understanding of physical reality. It has also, however, produced a great deal of confusion. For one thing, SI has created a profound rift in our knowledge of physical reality. As a result of its rise, our knowledge has become divided into two very different parts-classical and non-classical physics; so different, in fact, that one often gets the impression that we are dealing with two different, if not totally incommensurable, realities. Perhaps nothing illustrates this difference better than visualizability. Classical physics frequently uses images of objects-balls, bricks, planets, galaxies, etc.--that we envision moving and interacting with each other. Non-classical physics is all about mathematical formulas and equations that describe processes and effects that are impossible to visualize. In Erwin Schrödinger's apt description, QT “a formal theory of frightening, indeed repulsive, abstractness and lack of visualizability" [1] (p. 681).

The lack of visualizability, to which Schrödinger points, is not a whim of the framers of SI; it is due to the main innovation that SI has made. One of the principal novelties in SI is the recognition of the agency of the observer. According to SI, we cannot observe physical reality in isolation. When we conduct observations, we act upon reality and our actions produce effect. As Bohr has stressed: “... when searching for harmony in life one must never forget that in the drama of existence we are ourselves both actors and spectators" [2] (p. 236). Quantum reality manifests itself by responding to actions by the observer. So, what in the final analysis the observer observes is not reality as it is, but as it manifests itself in interactions with the observer and the instruments selected for observation. Strictly speaking, SI does not deal with individual particles. It assigns individuality to interactions. According to Bohr,

... the individuality of the typical quantum effects finds its proper expression in the circumstance that any attempt of subdividing the phenomena will demand a change in the experimental arrangement introducing new possibilities of interactions between objects and 
measuring instruments that in principle cannot be controlled [2] (p. 210).

And elsewhere:

... in quantum mechanics, we are not dealing with an arbitrary renunciation of a more detailed analysis of atomic phenomena, but with a recognition that such an analysis is in principle excluded [2] (p. 235).

In other words, SI tells us that we observe the interaction, made possible by mediation, between reality, on one hand, and the observer and the instruments that the observer chooses to use, on the other. This approach basically recognizes that mediation is necessary for the production of knowledge. Such mediation involves both the instruments chosen and the interpretation of results by the observer.

This approach has had a profound impact on research methodology. SI guides researchers to deal strictly with the experimental results that they observe, rather than reflect on reality that they, according to SI, cannot in principle observe. In the light of SI, such reflections are but idle speculations. When quantum experiments produce what from the common sense view appears as paradoxes, we should accept these paradoxes as given, no further questions asked.

Paradoxes create conflicting images that defy our imagination and are impossible to visualize. Indeed, how do you picture such well-known quantum phenomena as entanglement, superposition, or wave function? Moreover, the framers of SI have specifically forewarned us against making any attempts to envision quantum processes. Richard Feynman, a renowned physicist and a recipient of a Nobel Prize for his achievements in quantum physics, cautioned:

Do not keep saying to yourself, if you can possible avoid it, "But how can it be like that?" because you will get 'down the drain,' into a blind alley from which nobody has yet escaped. Nobody knows how it can be like that [3] (p. 129).

Since SI does not allow any probing beyond the phenomena accessible to observation, those who subscribe to SI must accept paradoxes and reject visualizability. SI guides researchers to practice instrumental approach and rely on formidable mathematical formalism that allows them to calculate and predict results of quantum experiments. Summarizing his understanding of the achievement of SI, Werner Heisenberg, one of the principal framers of this perspective, wrote:

The conception of the objective reality of the elementary particles has thus evaporated not into the cloud of some obscure new reality concept, but into the transparent clarity of mathematics that 
represents no longer the behavior of the particles but rather our knowledge of this behavior [4] (p. 2).

Mathematical formalism expunged visualizability from quantum mechanics. "Shut up and calculate" has become the most widely applied rule among the practitioners of SI.

As has already been indicated, the rejection of visualizability has become one of the markers of the dominant interpretation of QT. As has already been mentioned, this fact has left the system of our knowledge of physical reality deeply divided into two parts: classical and non-classical physics. The continued dominance of SI perpetuates this division. The perpetuation of the division hinders the equilibration of two parts (classical and non-classical) of the system of our knowledge of physical reality. The equilibration of differences is the process that creates new and more powerful levels of organization, which is essential for the evolution of systems, including systems of knowledge. The failure to equilibrate our knowledge of physical reality is one important factor that explains why no major fundamental ideas appeared in physics since the creation of SI. It also explains the repeated attempts to create alternative interpretations that challenge SI's dominance.

There is another reason why SI spurs the creation of new interpretations. As has been indicated earlier, SI recognizes the agency of the observer. According to this interpretation, we cannot in principle observe reality by itself but only the way it manifests itself in interactions with the instruments selected by the observer. In other words, what the observer observes is not reality by itself but only the phenomenal level of interactions. Thus SI formalism describes interactions between reality and the observer, not reality itself, since it is only this level that is accessible to the observer in experiments. The bizarre quantum phenomena that mathematical formalism describes appear on the phenomenal level of interactions-the only level accessible to observation. Therefore, strictly speaking, the conclusions that SI draws from observations of the phenomenal level cannot be extended to reality as it is; such extension is unwarranted by the theory itself.

When SI observes uncertainty on the phenomenal level of interactions, it cannot really extrapolate from these observations that reality is uncertain. According to its own premises, the only correct conclusion that it can make is that we do not know how reality is since uncertainty is observed only on the phenomenal level. We cannot assume that observing the manifestation of reality is the same as observing reality itself. Therefore, SI does not warrant the conclusion about uncertainty of reality that is accepted by the proponents of SI unquestionably and without any qualifications.

Since strictly speaking quantum experiments do not warrant any conclusions about the nature of reality, the key proposition that SI makes about the inherent uncertainty of physical reality can only be accepted as an axiom. However, in order to be accepted as a foundational proposition, it must be justified; otherwise, we can 
only see such proposition as subjective and arbitrary. SI does not provide such justification. Moreover, such justification is in principle impossible.

According to the proof provided by Austrian mathematician and logician Kurt Gödel, axiomatic systems can never establish their own consistency; in other words, they cannot prove that they do not contain statements that contradict its axioms. According to Gödel, any axiomatic system can have true sentences whose truth cannot be demonstrated by using the system's axioms; that is, they are not consistent with these axioms. In order to demonstrate their truth, one should resort to meta-mathematical procedures and construct a new and broader axiomatic structure that would be powerful enough to make such proof possible. However, as Gödel has made clear, even the new and enlarged structure will not be able to escape the same paradox as it will also allow other true sentences that are also indemonstrable [5].

Many computer scientists have argued in the same way that true uncertainty may exist but its existence cannot be demonstrated logically since meta-procedures required for such demonstration will necessarily reveal inconsistencies that contradict the original axiom. Hans Primas, for example, shows that if axiomatic principle, such as determinism, cannot be satisfied, "it can be enforced by choosing a larger state space [6] (p. 10)." Such enforcement, according to Primas, is perfectly compatible with mathematical probability theory because:

Every mathematically formulated dynamics of statistically reproducible events can be extended to a description in terms of a one-parameter group of automorphisms on an enlarged mathematical structure which describes a fictitious hidden determinism. Consequently, randomness in the sense of mathematical probability theory is only a weak generalization of determinism [6] (p. 1).

Jean Bricmont also sees ontic determinism lurking behind the appearance of quantum randomness [6, 7].

There is only one conclusion one can draw from the above discussion. SI does not and cannot provide either empirical evidence or logical justification for its foundational proposition about reality. It is impossible to prove that reality is uncertain. Thus, SI may be true but it is impossible to prove that it is true. In the absence of such proof-either empirical or logical-we have no choice but to view SI as subjective and arbitrary. In other words, SI cannot and does not produce a definitive interpretation of quantum mechanics. This fact and also the need to solve the problem of the division in the system of our knowledge of physical reality-so that the system could further evolve--justifies and legitimates efforts to construct interpretations based on different foundational propositions that rival SI.

\section{Bohmian Mechanics}


There are many interpretations that challenge the dominant position of SI. They include Bohmian mechanics, the pilot-wave theory, the hidden variable interpretation, the many-worlds interpretation, the many-minds interpretation, the consistent histories approach, and some others $[8,9,10,11]$. However, despite the growing variety of new versions, SI still retains its commanding presence and continues to define the work of researchers in quantum physics. Some critics explain these failures by the power of scientific establishment that backs SI and controls resources necessary for developing these alternative interpretations. Indeed the power of scientific establishment is considerable. It controls much of research funding, access to publications, the power of appointments and tenure decisions, etc.; and it certainly uses this power to support the theoretical perspective it has chosen to back up. In his famous book The Structure of Scientific Revolution, Thomas Kuhn points out a high degree of inertia in our scientific community. Many other researchers have expressed similar concerns $[12,13,14$, $15,16,17,18]$. However, force alone cannot explain the enduring dominance of SI. In order to explain the failure of alternative versions to open new vistas in studying physical reality, one should take a closer look at the challengers. Examining these failures is certainly important for making progress.

It is beyond the scope of this article to provide a detailed analysis of all theoretical perspectives that offer alternatives to SI. Such analysis requires a separate study. This paper will focus only on one interpretation that has attracted more attention than others. It is what has been dubbed as Bohmian mechanics named after its creator, American physicist David Bohm.

Bohm first formulated his theory back in the early 1950 [20]. Initially, the theory was quite successful and was able to gain some support in the physics community. However, as time passed, its influence waned and has only recently experienced some revival $[20,11]$.

Bohm certainly accepts the most important contribution of SI-its mathematical formalism. In contrast to SI, however, in Bohmian mechanics this formalism is more than just a convenient way to calculate and predict outcomes of quantum experiments. The phenomena that quantum equations formalize are, for Bohm, the actual aspects of reality. Bohmian mechanics explains even the weirdest quantum phenomena-such as non-locality, superposition, and backward causality-in terms of causes and effects. In Bohm's theoretical perspective, for example, non-localitya quantum phenomenon that involves speeds faster than the speed of light-is not merely a mathematical representation of some results of quantum experiments. It is, for Bohm, a fundamental property of reality. The famous wave function is not just a convenient formula for calculating outcomes of quantum experiments; in the Bohmian world it actually guides particles and determines their state.

Quantum formalism is essentially mathematical equations of motion that, like all other mathematical equations, obey the logic of mathematics. Viewing this 
mathematical formalism as more than just the way of calculating and predicting the outcomes of the phenomena it describes, but as representing the actual aspects of quantum reality, entails one inevitable consequence. In order to conserve the logical necessity of mathematical equations, Bohm has to recognize determinism as the fundamental property of reality. Thus, in contrast to SI that sees uncertainty as a fundamental property of reality, Bohmian mechanics recognizes that quantum reality as fully determinate. Indeed, Bohm acknowledges the existence of uncertainty, but this uncertainty is strictly epistemological, that is, it is due to our lack of knowledge about reality, rather than the way this reality is $[20,11]$.

One can see from the above that Bohm completely and radically reverses SI. The strategy of reversal results in several consequences that do not work in favor of the Bohmian alternative. As a mere inversion of SI, Bohmian mechanics has the same explanatory power as SI. Like SI, Bohm also essentially interprets, or rather reinterprets, the phenomena that can be observed on the level of interactions. When he accepts these phenomena as fundamental properties of physical reality, he commits the same logical mistake by attributing to reality what essentially belongs to the phenomenal level, which is unwarranted. We certainly cannot view the manifestations of reality on the level of phenomena as the actual and real properties of reality by itself. Since Bohm's fundamental proposition about the determinate nature of reality is derived from mathematical formalism, we must view it as an axiomatic proposition that Bohm uses to organize his theoretical perspective. However, he also does not provide logical justification for this claim. Moreover, there can be no proof for ontic determinism. Just like the existence of uncertainty and randomness is impossible to prove, their existence is also impossible to disprove, which undermines any claim of ontic determinism: if there is a possibility that reality can be uncertain, then the claim that reality is determinate must be in doubt.

Jean Bricmont simply dismisses the entire issue of the intrinsic nature of determinism as ultimately irrelevant. Bricmont examines two current definitions of determinism. He finds that one definition in which determinism is conflated with predictability renders determinism trivially false. Considering the other definition that avoids the conflation, Bricmont asks a pertinent question: is there a function-in a Platonic sense (that is, independent of our ignorance)--that determines a finite sequence of sets of numbers that never repeats itself in a unique way. His answer is that the existence of such function is simply impossible to disprove because one can always find a function or even many functions that map "each set into the next one" [7] (p. 2). Bricmont's conclusion dismisses the whole issue of determinism as utterly irrelevant to science. In his view, "there is no notion of determinism that would make the question [of determinism] scientifically relevant ... ontically it [determinism] is true but uninteresting [that is, impossible to disprove]" [7] (p.2). "I don't know," he adds, "how to formulate the issue of determinism so that the question becomes interesting" [7] (p. 1). The point is that either determinism or uncertainty may actually exist in the ontic sense, but this existence is impossible to 
prove; and if the claim that reality is either uncertain or determinate cannot be justified, any theory based on either of these claims cannot be accepted as definitive.

In a practical sense, Bohmian determinate mechanics also offers no advantages in comparison with SI. While its determinism suggests that since all particles in the universe have a determinate position, we can calculate their positions at any moment if we know their initial position and the wave function of the universe. In practice, however, the acquisition of such knowledge encounters an insurmountable problem. In order to perform such calculations, one should know the initial position of particles, including particles in the brain of the observer and their wave function. Observing particles in one's own brain requires constructing a position from which such observation can be conducted. However, the problem is that such position should also be accessible to observing, which will inevitable lead to the problem that Niklas Luhman defined as infinite regress that inevitably results from observing the observing. Bohmian mechanics does not resolve this problem. In principle, we can know the position of every particle in the universe; in practice, however, such knowledge is impossible [11] [22] (p. 479) [23] (p. 329).

In contrast to SI, Bohmian mechanics is visualizable. But that is probably the only significant difference between the two interpretations. Just like SI, Bohm's perspective also rests on the shaky foundation of a subjective and arbitrary axiomatic proposition. It offers few, if any, practical advantages in comparison with SI. As a result, one cannot accept Bohmian mechanics as a definitive interpretation of quantum theory. Just like SI, it offers nothing that can help integrate classical and non-classical physics and, consequently, does not contribute to the further evolution of the system of our knowledge of physical reality. Due to these reasons, Bohmian mechanics fails to present a real and constructive alternative to SI.

\section{The Solution}

As has been indicated earlier, the division in the system of our knowledge of physical reality creates a problem. It prevents this system from evolving and transcending the boundaries of the existing knowledge. The achievement of this goal requires the creation of a new perspective that would be broad enough to include both classical and non-classical physics as its two specific cased-that is, cases that are true under special circumstances or assumptions. How can we solve this problem of division and create such broader perspective? Ironically, SI suggests a solution but it fails to follow through on its own suggestion.

SI claims that the observer has access only to interactions between quantum reality and the instruments that the observer chooses to conduct observations. So, what is this interaction and what is its nature?

According to SI, observations affect the objects that we observe. In other words, it changes these objects in some way. Therefore, the act of observing is a creative act that changes reality. 
However, there is more to this interaction. The objects of observation are not passive. They also respond to the action by the observer and affect the outcome of interactions. Therefore, the response of objects to observing changes the outcome and, consequently, also represents a creative act.

The conclusion that follows from the above considerations is that the process of interaction that takes place between the object and the observer (subject) is a creative act. SI is absolutely correct: the only reality available to us is the process of creation. This process creates our knowledge.

We are part of the universe. Like any other object in our universe we interact with other objects. Hence we are fully justified in generalizing our interactions with objects we observe to all other interactions. Therefore, interactions with objects we observe are a subset of a more general type of interactions that take place among all objects in our universe. It is a universal process of interactions and this process is creative. It creates objects in our universe and our universe as a whole.

The main property of our universe is its uniqueness: it is all there is. There is nothing outside it; in fact, there is no outside. As there is nothing outside our universe, nothing can come into it and nothing can disappear from it because there is nowhere to disappear. Consequently, everything must be conserved.

Conservation is fundamental to our universe; it originates in the uniqueness of our universe and it gives rise to the process of creation.

Our universe contains an enormous variety of different forms. All these forms are finite; and the resources for sustaining finite forms are also finite. In order to conserve finite forms, their range of possibilities, or degrees of freedom, must expand, which will allow them access to new resources. Gaining new possibilities requires new properties, that is, properties that have had no prior existence; in other words, it requires an act of creation. New properties can arise only as a result of the integration of differences that leads to the emergence of the new and more powerful levels of organization. Conservation is impossible without the process of creating such new and more powerful levels of organization. If a system does not evolve, it begins to disintegrate.

We humans are also creations of this process. As its creations we are also part of this process. We have inherited from this process our capacity to create. Over the course of our history we have demonstrated this capacity in works of art, in science and technology, in organizing our society and its institutions. Our civilization itself is a remarkable evidence of our creativity. It is the source of our power and prosperity.

Given the importance of the process of creation in our life, one would expect that we would use it as the main organizing principle of our practice. Yet odd as it may seem, this process is not central to our civilization. Our civilization is decidedly 
anthropocentric. Since at least the Enlightenment, if not earlier, we have made the human subject the main organizing principle of our civilization, its practice and institutions.

This article has shown that the adoption of subjectivity as the main organizing principle of our mental universe is essentially a subjective and arbitrary act-it is not supported either by justification or by experimental verification. For this reason, it cannot and does not provide us with a definitive way of interpreting reality. It generates confusion, inconsistencies and paradoxes that hinder the progress of our civilization and create many current problems that we as a civilization face today, including the stagnation of our physical science that remains split between two separate and rather incommensurable, despite some similarities, conceptions of reality.

As this article suggests, the solution of the problem that plagues the system of our knowledge of physical reality is in creating the frame for a new theoretical perspective that would be able to integrate both classical and non-classical physics as its particular cases; that is, cases that are true under special circumstances or axioms. Such frame will require the foundation that is capable of including axiomatic propositions upon which both classical and non-classical physics rest.

The process of creation can serve as the basis for such integration. The foundational proposition for classical physics is the centrality of the object. By contrast, non-classical physics is organized around the recognition of the centrality of the subject and subjectivity. This article agrees with SI that we cannot observe quantum reality as it is; we can only access the interaction between this reality and the observational instruments chosen by the subject. This article has shown that the process of creation is the essence of this interaction, and this process is ultimately the source of everything that exists in our universe. Therefore, this process can serve as a foundational proposition that integrates subjectivity and the object. A theoretical perspective constructed on this foundation can integrate classical and non-classical physics. Therefore, we should adopt the process of creation as the main focus of our science practice.

What will such new science practice involve? It is beyond the scope of this article to provide a detailed answer to this question; such answer has been provided elsewhere [21]. However, an outline of the main points may be in order.

First of all, we must recognize that the main motive of our intellectual activity is not knowledge. Knowledge is merely a by-product of this activity. Its true motivation is the construction of new and increasingly more powerful levels of our mental organization as a way of conserving our mental constructions. These new and more powerful levels of organization will allow us to establish new one-to-one correspondences between our mental constructs and reality, thus generating new knowledge.

Since the construction of new and more powerful levels of organization involves integrating differences, the most important form of validation of new knowledge is its 
inclusiveness and comprehensiveness. This does not mean that experimental support is not necessary. Our mental activity emerges from practical activity. Therefore, this process should allow also the inversion from theoretical constructs to practice. However, mental constructs are the most powerful forms of organization of reality and they can always create one-to-one correspondences with physical reality and such correspondences alone cannot validate knowledge.

This approach follows from the understanding that the process of creation is the source of our knowledge and, in fact, the source of everything that exists in our universe. By understanding this process and applying this knowledge to our practice we will place our practice on the solid foundation of our reason and rationality and will no longer rely on domination and force in determining what constitutes knowledge and what does not.

\section{Conclusion}

Over fifty years ago Thomas Kuhn in his now famous book The Structure of Scientific Revolutions addressed the issue of stagnation in the evolution of our knowledge [12]. It is in many ways a pessimistic book. It recognizes that our science community has a tendency to succumb to inertia that leads to long periods of stagnation. Moreover, the author suggests that such stagnation is inevitable and there is nothing that we can do to ameliorate this condition. He does not believe that human reason and rationality are capable of overcoming this inertia and prevent it from hindering the progress of our knowledge. In a way, he suggests a very anti-Enlightenment conclusion that human reason cannot be the arbiter in our practice, including our science practice. According to Kuhn, only death and destruction can ultimately settle the conflict between stasis and change, between old ways and innovation. The old generation should die out so that the new one could pave the way for new ideas and theories. But even death and destruction may not necessarily be a guarantee against inertia and stagnation, and assure the ascension of new ideas. The modern tools of indoctrination and thought control may be powerful enough to prevent the rise of a new generation and the spread of new ideas. Inertia and stagnation may very well become an inescapable condition of our civilization.

In a way Kuhn tells us that we are the source of our progress. For this reason, the only guarantee we can have against stagnation is our full commitment to a science practice that is based on reason and rationality-- not domination and force, no matter how benevolent the motivation behind them may be. We need to understand the process that plays a critical role in knowledge production-the process that is fundamental to the existence of our universe and that we have inherited in the course of a long and arduous evolution. We need to place our hope in the capacity of our reason to understand even this fundamental process that transcends our own existence; and we must work hard to realize this hope.

Conflict of Interests: The author declares no conflict of interests. 


\section{References}

1. Cramer, J. G. The Transactional Interpretation of Quantum Mechanics. Reviews of Modern Physics 1986, 58 (3), 647-87.

2. Bohr, N. Discussion with Einstein on Epistemological Problems of Atomic Physics. In Albert Einstein: Philosopher-Scientists; Schilpp, P. A. Ed.; Open Court: La Salle, Ill., 1969.

3. Feynman, R. The Character of Physical Law; The M.I.T. Press: Cambridge, 1985.

4. Stapp, H. P. Quantum Theory and the Role of Mind in Nature; ArXiv:QuantPh/0103043, March 9, 2001. http://arxiv.org/abs/quant-ph/0103043.

5. Nagel E. and James R. Newman, J. R. Gödel's Proof; University Press: New York, 1953.

6. Primas, H. Hidden Determinism, Probability, and Time's Arrow. In Between Chance and Choice: Interdisciplinary Perspectives on Determinism; Atmanspacher $\mathrm{H}$. andR. Bishop, R., Eds.; Imprint Academic: Thorverton, 2002).

7. Bricmont, J. Determinism, Chaos, and Quantum Mechanics; http://www.scribd.com/doc/11328575/Jean-Bricmont-Determinism-Chaos-andQuantum-Mechanics (accessed June 22, 2010);

8. Ballentine, L. E. Quantum Mechanics: A Modern Development; World Scientific: River Edge, NJ, 2014.

9. Putnam, H. A Philosopher Looks at Quantum Mechanics (Again). The British Journal for the Philosophy of Science, 2005, 56 (4), 615-634.

10. Belavkin, V. P. Quantum Probabilities and Paradoxes of the Quantum Century, 2005. http://arxiv.org/abs/math.PR/0512415;

11. Albert, D. Z. Bohm's Alternative to Quantum Mechanics. Scientific American May 1994, 56-67.

12. Kuhn, T. S. The Structure of Scientific Revolutions; Uiversity of Chicago Press: Chicago, 2012.

13. Weiler, H. N. Whose Knowledge Matters? Development and the Politics of Knowledge. In Entwicklung Als Beruf; edited by Theodor Hanf T., Weiler H. N., and Dickow H.; Eds.; Nomos: Baden-Baden, 2009. 
14. Araya, D. Cultural Democracy: Universities in the Creative Economy. Policy Futures in Education 2010; 8 (2), 217-32.

15. Latour, B. Politics of Nature: How to Bring the Sciences into Democracy; Harvard University Press: Cambridge, Mass, 2004.

16. Martin Lopez-Corredoira, M. Sociology of modern cosmology 2008; http://arxiv.org/abs/0812.0537 (accessed 17 July 17, 2013).

17. Brown, R. H. Modern Science and Its Critics: Toward a Post-Positivist Legitimization of Science. New Literary History 1998; 29 (3), 521-50.

18. Charlton, B. G. Why Are Modern Scientists so Dull? How Science Selects for Perseverance and Sociability at the Expense of Intelligence and Creativity. Medical Hypotheses 2009; 72 (3), 237-43.

19. Shkliarevsky, G. Science and Its Discontents: Is There an End to Knowing? Systems Research and Behavioral Science; 2013; 30 (1), 43-55.

20. Bohm, D. A Suggested Interpretation of the Quantum Theory in Terms of 'Hidden' Variables. I. Physical Review 1952; 85 (2), 166-79.

21. Shkliarevsky, G. The Civilization at a Crossroads: Constructing the Paradigm Shift; Glasstree Publishing: Raleigh, NC, 2017.

22. Luhmann, N. Social Systems; Stanford University Press: Stanford, 1995.

23. Shkliarevsky, G. The Paradox of Observing, Autopoiesis, and the Future of Social Sciences. Systems Research and Behavioral Science 2007; 24 (3), 323-32. 